\title{
Astronomia indígena na formação de professores: uma possibilidade a partir da abordagem CTS
}

\author{
Astronomy in indigenous teacher training: a chance from the approach CTs \\ Luciana Flôr Correa ${ }^{1}$ e Bruno dos Santos Simões ${ }^{2}$
1Doutoranda em Educação Científica e Tecnológica. Bolsista FUMDES/SC.
Universidade Federal de Santa Catarina, SC, Brasil
luciana.Flor@unisul.br
${ }^{2}$ Doutorando em Educação Científica e Tecnológica. Bolsista do CNPq.
Universidade Federal de Santa Catarina, SC, Brasil
simoes89@hotmail.com

\begin{abstract}
Resumo
Este trabalho tem por objetivo discutir a valorização da cultura científica dos povos indígenas, bem como a importância dessa cultura para a promoção do pensamento crítico por parte dos estudantes e professores em formação. Para tanto, argumentamos em prol da etnoastronomia, um conhecimento desenvolvido por povos nativos das Américas e que apresenta riqueza de informações, cultura e conhecimento tácito. Nossa discussão vai nesse sentido por entendermos a potencialidade pedagógica que a etnoastronomia proporciona, por se tratar de uma astronomia baseada em elementos sensoriais, e não em elementos geométricos e abstratos, e também por fazer alusão a elementos da nossa natureza (sobretudo fauna e flora) e história, promovendo autoestima e valorização dos saberes antigos, salientando que as diferentes interpretações da mesma região do céu, feitas por diversas culturas, auxiliam na compreensão das diversidades culturais. Dessa forma, para além de buscar a inserção de conteúdos de Astronomia na escola básica, também devemos buscar a valorização e o ensino da etnoastronomia; que pode ser realizada e otimizada se articulada à abordagem Ciência, Tecnologia e Sociedade (CTS).
\end{abstract}

Palavras-chave: Etnociência. Astronomia Indígena. Ciência. Tecnologia e Sociedade. Formação de Professores.

\begin{abstract}
This work aims to discuss the development of a scientific culture of indigenous peoples, as well as the importance of culture for the promotion of critical thinking by students and teachers in training. Therefore, we argue in favor of ethnoastronomy, knowledge developed by indigenous peoples of the Americas and presenting a wealth of information, culture and tacit knowledge. Our discussion will do so by understanding the pedagogical potential that ethnoastronomy provides, for it is an astronomy based on sensory elements, not geometric and abstract elements, and also to allude to elements of our nature (mainly fauna and flora) and history, promoting self-esteem and appreciation of ancient knowledge, stressing that different interpretations of the same region of the sky, made by diverse cultures, help in understanding cultural diversity. Thus, in addition to seeking the inclusion of Astronomy contents in elementary school, we must also pursue recovery and the teaching of ethnoastronomy; which can be realized and optimized if articulated approach to Science, Technology and Society (STS). Abstract here Abstract here Abstract here Abstract here Abstract here Abstract here
\end{abstract}

Keywords: Ethnoscience. Indigenous astronomy. Science, Technology. and Society. Teacher training. 


\section{Introdução}

Os temas relacionados com os saberes e tecnologias populares, locais, tradicionais, nativas e indígenas nos currículos de ciências têm sido considerados por vários estudiosos em diferentes contextos (CHASSOT, 2001, 2007; FRANCISCO, 2005; PINHEIRO; GIORDAN, 2010). O que tem se destacado nessas experiências é que o processo educativo estendese para além das experiências de aprendizagem formal, em laboratório ou sala de aula, implicando aumento da confiança dos alunos com base no reconhecimento de suas origens e no respeito pelos saberes de seus antepassados.

Outros autores também vêm apontando benefícios em práticas dessa natureza. Pomeroy (1994) citado por Pinheiro e Giordan (2010), por exemplo, menciona o aumento do interesse dos alunos pela ciência ao vê-la sendo investigada em contextos de produção de conhecimento popular ou nas tecnologias nativas. Chassot (2007) indica a possibilidade de compreender melhor a história da sociedade e o papel da ciência e da tecnologia na vida moderna pelos jovens no estudo de saberes populares na escola. Cobern e Loving (2001) sugerem a possibilidade de se compreender melhor a natureza da ciência na interação com outros modos de conhecer. George (1992) menciona a motivação, a participação ativa dos alunos, o elevado nível de socialização nas aulas, o melhor desempenho dos alunos, a compreensão mais rápida e melhor dos conceitos científicos e a ampliação da visão de ciência e sua aplicação na vida, sem, contudo, deixar de mencionar também algumas desvantagens, tais como o desconhecimento pelos professores dos princípios científicos operantes em alguns saberes e práticas nativas e a necessidade de haver formação específica e mudanças na prática pedagógica.

Além disso, conforme indicam Pinheiro e Giordan (2010), a análise das discussões no âmbito da educação multicultural em ciências também nos permite identificar o contato dos alunos com outras epistemologias, o que nos faz entender ser fundamental estabelecer conhecimento científico sobre esses saberes e analisar suas formas de inserção em sala de aula e de promoção de diálogos com a ciência ensinada na escola.
Diante disso, esse é um tema bastante apropriado para, articulado à perspectiva Ciência, Tecnologia e Sociedade (CTS), constar nos currículos escolares e nos cursos de formação de professores, haja vista que há nele um componente de consequências sociais, oriundo da tradição versus tecnologias modernas, que são envoltas em interesses econômicos, industriais, éticos, étnicos, científicos, sociais, dentre outros, o que acarreta um grande número de visões e de ângulos de análise para a situação.

Santos e Mortimer (2002) citam Hofstein et al. (1988) e argumentam que o enfoque CTS pode ser caracterizado como o ensino do conteúdo de ciências no contexto autêntico do seu meio tecnológico e social, em que os estudantes agregam o conhecimento científico com a tecnologia e o mundo social de suas experiências do cotidiano. A proposta curricular de CTS corresponderia, portanto, a uma integração entre educação científica, tecnológica e social, em que os conteúdos científicos e tecnológicos são estudados juntamente com a discussão de seus aspectos históricos, éticos, políticos e socioeconômicos (LÓPEZ; CEREZO, 1996 apud SANTOS; MORTIMER, 2002).

Sob esta ótica compreendemos que a discussão da etnociência, e mais especificamente da astronomia indígena, além de contemporânea, valoriza a cultura e produção de conhecimento dos povos indígenas de nosso país, além de encontra-se retratada nos Parâmetros Curriculares Nacionais (PCNs), no que tange às Ciências Naturais, e permitirá, num futuro próximo, discussões representativas de diversos setores e segmentos da sociedade brasileira.

Nesse sentido, não é nosso objetivo aqui julgar o certo ou o errado, mas promover o exercício da controvérsia, da qual deve surgir o exercício da reflexão em torno de estruturas etnocientíficas e a construção da aprendizagem e da convivência de grupos com ideias e posições distintas, sobre o qual deve se estruturar uma sociedade democrática.

Como o enfoque CTS trilha um caminho alternativo, iremos discutir a ciência como uma construção histórica e cultural humana, não como uma forma de atingir a verdade plena e soberana. Por isso, nada melhor do que apresentar novas ciências, novas verdades 
construídas sobre outras realidades e maneiras de ver e sentir o mundo.

Além disso, acreditamos que lançar mão da estratégia de mostrar para o público em formação e de futuros formadores um pouco da construção de conhecimento de etnias distintas pode gerar reflexões mais pertinentes sobre o tema.

Segundo Lima (2000, p. 418), é válido e importante

[...] gerar a inquietação por um saber menos totalizante em sua aparência e mais libertário em seus efeitos do que o ensinado pelo Estado nacional, permitir crescimento do desejo da diferença e a descrenças nas verdades oficiais, conquanto sabendo-se que temos de lidar com elas, produzir elementos para consciências questionadoras.

Dentre as ciências, escolhemos trabalhar com as ciências indígenas, pois acreditamos que existe uma dívida histórica por parte do EstadoNação em respeitar e valorizar esses povos, principalmente em reconhecer a individualidade de suas culturas e maneiras diversas e específicas de se relacionarem com o mundo, não negando esse direito às demais etnias que igualmente foram relegadas e subjugadas (FONSECA; PINTO; JURBERG, 2007).

Diante desse contexto, acreditamos agregar grande possibilidade de sucesso com a articulação entre a abordagem CTS e a etnoastronomia, a fim de exemplificar para a comunidade escolar outra forma de construção e de convivência científica, já que o grau de interesse que este tema desperta é relevante.

\section{Breves apontamentos sobre a etnociência}

Pinheiro e Giordan (2010) comentam que uma visão comum sobre etnociência costuma associála ao "conhecimento científico desvendado" nos saberes e práticas de grupos culturais específicos, como os indígenas, por exemplo. No entanto, Pinheiro e Giordan (2010), citando Pomeroy (1994), trazem outra direção ao dizer que no domínio das etnociências deve haver evidências da existência de práticas científicas entre os povos nativos, seja na astronomia, na ecologia, na agricultura ou nas práticas de caça. Os autores complementam argumentando que a etnociência foi associada a "métodos, processos de pensamento, operações mentais, valores, conceitos e experiências através das quais os grupos Americanos Nativos compreendem, refletem e obtêm conhecimento empírico sobre o mundo natural" (PINHEIRO; GIORDAN, 2010, p. 360).

De fato, em suas origens, a etnociência foi associada ao conhecimento indígena sobre a Natureza, sendo uma denominação atribuída por cientistas envolvidos com pesquisas junto a essas comunidades (PINHEIRO; GIORDAN, 2010). Os autores comentam ainda que:

Em um artigo publicado em 1974, Sturtevant apresentou a etnociência como a Nova Etnografia (the New Ethnography), mas não considerou adequada a denominação etnociência por duas razões: primeiro por sugerir que outros tipos de etnografia não sejam ciência e segundo por admitir que as taxonomias populares sejam ciência. A ciência aqui se associava à classificação ou ordenação das coisas em classes (taxonomia) pelos indígenas, sendo vista como o grau de redução do caos por uma dada sociedade (etnociência clássica) (PINHEIRO; GIORDAN, 2010, p. 360).

O prefixo etno, por sua vez, foi inicialmente definido como o que é típico de uma dada cultura ou grupo social. A etnobotânica, por exemplo, seria uma concepção cultural específica do universo dos vegetais, que poderia ou não estar relacionada à taxonomia da botânica oficial. Hoje tal prefixo é aceito como sendo mais amplo e inclui considerações como linguagem, jargões, códigos de comportamento, mitos e símbolos próprios de um contexto cultural (PINHEIRO; GIORDAN, 2010, p. 360).

Com o desenvolvimento da sociolinguística e da ethnoscience foi surgindo uma enormidade de termos disciplinares que buscavam identificar aspectos científicos significantes nas comunidades investigadas. Essas várias áreas (denominadas de etno- $X$ ), no entanto, não tinham uma relação específica com o campo da educação científica, em particular. Eram estudos que buscavam essencialmente investigar as populações tradicionais tentando compreender seus conhecimentos sobre o mundo natural, as taxonomias e as classificações totais (PINHEIRO; GIORDAN, 2010).

Uma etno- $X$ que surgiu essencialmente compromissada com a educação foi a etnomatemática, que teve sua origem entre pesquisadores brasileiros e que atualmente abarca pesquisadores, estudos e experiências 
educacionais em todo o mundo (SILVA, 2011). Outras etno-X que têm se desenvolvido no Brasil e em outras partes do mundo, com sociedades acadêmicas organizadas, são a etnobiologia (TRÉZ, 2011) e a etnoastronomia (AFONSO, s/d; AFONSO, 2009).

Diferente das etno- $X$ mencionadas acima, a Etnofísica e a Etnoquímica ainda são áreas de saber incipientes. Em nosso conhecimento, o primeiro estudo que faz menção a elas é uma coletânea de reflexões e pesquisas elaborada por pesquisadores do Instituto Superior Pedagógico, em Moçambique (PINHEIRO; GIORDAN, 2010).

Então, dentre as etnociências mencionadas acima, optamos por argumentar mais a respeito da etnoastronomia. Tanto por compartilharmos das concepções de Afonso (2009), de que a etnoastronomia possibilita o desenvolvimento de um pensamento crítico em relação à cultura e à ciência, quanto pelo fato de entendermos que essa herança cultural do nosso país deve ser preservada e valorizada, com o intuito de suscitar uma população consciente da riqueza cultural de nossa nação.

\section{Contextualizando a astronomia indígena}

No que concerne a astronomia indígena, Fares et al. (2004, p. 78) fazem um levantamento histórico ao qual chamam de construção social do céu. Nessa construção os autores comentam que os povos babilônicos da antiga Mesopotâmia são os primeiros responsáveis por grande parte do conhecimento que se tem hoje das constelações clássicas e dos seus respectivos mitos. Os babilônios, por sua vez, herdaram seu conhecimento astronômico dos povos sumérios, primeiros habitantes daquela região, dos quais são descendentes. Essa civilização suméria remonta a um período entre 4000 e 3000 anos a.C. e se destaca pelo alto grau de organização sócio-político-econômica.

A relação do homem com o céu ocorre por vários motivos, motivos esses que vão se alterando ao longo da história. Certamente, por um lado, há todo o encantamento e mistério, próprio de tudo que é grandioso e desconhecido, instigando a contemplação celeste; e, por outro, existe a necessidade de orientação, que para os povos antigos era fundamental à sobrevivência, visto não disporem de outros meios de localização no espaço e tempo que não fossem as estrelas (FARES et al., 2004).

Os mesmos autores ainda afirmam que,

(...) tal relação foi, inicialmente, reforçada com a sedentarização dos povos. Há cerca de 8000 anos o homem era caçador, mas começava a desenvolver uma agricultura rudimentar. Dependia, então, dos fenômenos sazonais para a prática da caça, devido à migração dos animais e devido às chuvas para a agricultura. [...] A orientação foi outra necessidade que motivou o estudo do céu, pois o homem deixara de ser nômade, tinha agora um lugar fixo para onde retornava após o período de caça. Utilizando as estrelas como guia, ele podia se afastar de seu lar na certeza de reencontrá-lo. Nesse momento, surge outra ideia, a de reunir as estrelas em grupos para facilitar o seu reconhecimento, dando origem, assim, às constelações. (FARES et al., 2004, p. 78).

Normalmente quando se fala em Astronomia, as pessoas tendem a lembrar-se dos povos gregos, em alguns casos dos árabes, babilônios, sumérios etc. Ou ainda das experiências realizadas por Galileu e sua luneta, ou por Kepler e seus cálculos complexos das órbitas celestes. Mas o que poucas pessoas lembram, ou mesmo sabem, é que vários outros povos espalhados pelo mundo também desenvolveram seus conhecimentos astronômicos. Podemos destacar, por exemplo, a Astronomia desenvolvida por tribos indígenas no Brasil. Afonso $(2009$, p. 1) ressalta esse fato afirmando que "os conhecimentos astronômicos empíricos dos indígenas, relativos aos movimentos do Sol, da Lua, da Via-Láctea e de suas constelações, associados à biodiversidade local, suficientes para a sobrevivência em sociedade, são desconhecidos por muitos historiadores da ciência".

Quando, em 1632, Galileu Galilei publicou o livro: "Diálogo sobre os dois máximos sistemas do mundo; ptolomaico e copernicano", ele afirmava que a principal causa do fenômeno das marés seriam os dois movimentos circulares da Terra: o de rotação em torno de seu eixo (diurno) e o de translação em torno do Sol (anual), desconsiderando a influência da Lua (AFONSO, 2009).

Mas, em 1687, Isaac Newton demonstrou que a causa das marés é a atração gravitacional do Sol e, principalmente, da Lua sobre a 
superfície da Terra, o que mostra que, muito antes da Teoria de Galileu, que, nesse aspecto, não considerava a Lua, os indígenas que habitavam o Brasil já sabiam que ela é a principal causadora das marés, conforme indicava d'Abbeville.

Em 1614, quando o monge capuchinho francês Claude d'Abbeville viveu um tempo com os tupinambás, no Maranhão, ele aprendeu muito sobre aquela cultura e escreveu sobre ela em alguns manuscritos. Afonso (2009) comenta que os indígenas já possuíam o conhecimento de que a Lua influencia o fluxo das marés. Também eram capazes de diferenciar o tipo de maré para a Lua cheia e as demais. Ao levar em consideração que, em 1632, Galileu Galilei publicou seu livro falando sobre o fenômeno das marés e atribuindo-o ao movimento da Terra, podemos afirmar que o conhecimento dos tupinambás era bem desenvolvido para atender às necessidades das tribos.

Os indígenas observavam os movimentos aparentes do Sol para determinar o meio dia solar, os pontos cardeais e as estações do ano, utilizando um instrumento chamado Gnômon, que consiste de uma haste cravada verticalmente no solo, da qual se observa a sombra projetada pelo Sol, sobre um terreno horizontal (AFONSO, 2009). Apesar de conhecer as fases da Lua e seus movimentos ao longo do ano, Claude d'Abbeville acreditava que os tupinambás utilizavam o Sol como um tipo de medidor dos dias do ano, ou seja, um calendário solar (LIMA; MOREIRA, 2005).

Em sua estadia no Maranhão, d'Abbeville pôde observar que os índios já conheciam e descreviam o movimento do Sol ao longo do ano e sabiam se com ele viria vento ou chuva; conheciam as estações do ano e controlavam o passar dos meses por meio das chuvas ou do ciclo do caju.

Além da orientação geográfica, um dos principais objetivos práticos da astronomia indígena era sua utilização na agricultura. Os indígenas associavam as estações do ano e as fases da Lua com a biodiversidade local, para determinarem a época de plantio e da colheita, bem como para a melhoria da produção e o controle natural das pragas. Eles consideram que a melhor época para certas atividades, tais como, a caça, o plantio e o corte de madeira, é perto da lua nova, pois perto da lua cheia os animais se tornam mais agitados devido ao aumento de luminosidade, por exemplo, a incidência dos percevejos que atacam a lavoura (AFONSO, 2009, p. 2).

Isso mostra o quão avançado era o sistema astronômico desses povos. $\mathrm{O}$ mais interessante é que esses conhecimentos não eram exclusividade dos tupinambás, haja vista que o povo guarani também possuía uma Astronomia bem desenvolvida.

Ao estudarem a obra de Claude d'Abbeville, Lima e Moreira (2005) trazem um quadro com diversas palavras e seus significados, muitas vezes bem extensos, que mostram a riqueza linguística dos tupinambás em relação à Astronomia.

Outro aspecto interessante da Astronomia indígena no Brasil é a identificação das constelações. Afonso $(2009$, p. 3$)$ destaca os três principais pontos que diferem a Astronomia indígena da ocidental tradicionalmente conhecida. São eles:

- Primeiro, as principais constelações ocidentais registradas pelos povos antigos são aquelas que interceptam o caminho imaginário que chamamos de eclíptica, por onde aparentemente passa o Sol, e próximo do qual encontramos a Lua e os planetas. Essas constelações são chamadas zodiacais. As principais constelações indígenas estão localizadas na Via Láctea, a faixa esbranquiçada que atravessa o céu, onde as estrelas e as nebulosas aparecem em maior quantidade, facilmente visível à noite.

- Segundo, os desenhos das constelações ocidentais são feitos pela união de estrelas. Mas, para os indígenas, as constelações são constituídas pela união de estrelas e, também, pelas manchas claras e escuras da Via Láctea, sendo mais fáceis de imaginar. Muitas vezes, apenas as manchas claras ou escuras, sem estrelas, formam uma constelação. A Grande Nuvem de Magalhães e a Pequena Nuvem de Magalhães são consideradas constelações.

- $\mathrm{O}$ terceiro aspecto que diferencia as constelações indígenas das ocidentais está relacionado ao número delas conhecido pelos indígenas. A União Astronômica Internacional (UAI) utiliza um total de 88 constelações, distribuídas nos dois hemisférios terrestres, enquanto certos grupos indígenas já nos mostraram mais de cem constelações, vistas de 
sua região de observação. Quando indagados sobre quantas constelações existem, os pajés dizem que tudo que existe no céu existe também na Terra, que nada mais seria do que uma cópia imperfeita do céu. Assim, cada animal terrestre tem seu correspondente celeste, em forma de constelação.

$\mathrm{Na}$ identificação indígena, por exemplo, Cruzeiro do Sul fica em plena Via Láctea, sendo a constelação mais conhecida dos indígenas do Hemisfério Sul, que a utilizam para determinar os pontos cardeais, as 4 estações do ano e a duração do tempo à noite. As Plêiades ficam em segundo lugar, sendo utilizadas para calendário (AFONSO, 2009).

Da mesma maneira, atualmente para os tembé, que habitam o norte do Brasil, o surgimento das Plêiades anuncia a estação da chuva e o seu ocaso: quando elas desaparecem no lado oeste, ao anoitecer, indica a estação da seca. Para os guarani do sul do país, o aparecimento das Plêiades anuncia o verão, enquanto $o$ seu desaparecimento indica a proximidade do inverno. Além dessas duas constelações, há outras que servem para calendário e orientação geográfica, tais como o Colibri, o Homem Velho, a Ema e a Cervo-doPantanal, todas elas localizadas na Via Láctea (AFONSO, s/d; AFONSO, 2009).

Nas figuras a seguir, podemos observar a região do espaço em que se localiza a Via Láctea. Na Figura 1 a denominação das constelações greco-romanas e na Figura 2 uma denominação indígena.

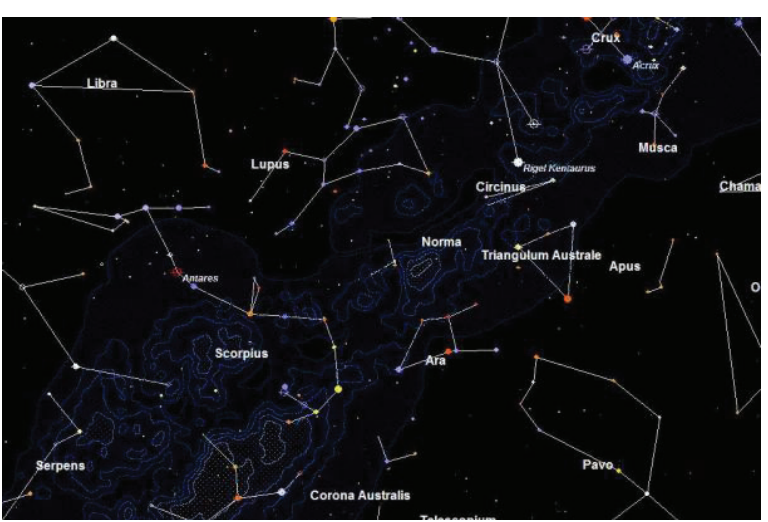

Figura 1 - Constelações greco-romanas Fonte: Afonso (s/d)

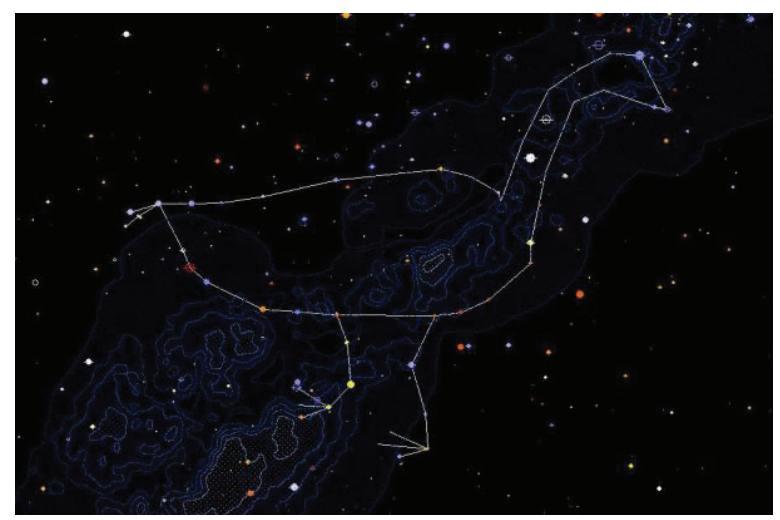

Figura 2 - Constelação da Ema

Fonte: Afonso (s/d)

Citando Barros (2004), Fares et al. (2004) dão outro exemplo de cultura astronômica indígena. Os autores citam o caso da comunidade dos tembé-tenetehara da aldeia Teko-Haw, localizada na área do alto rio Gurupi, na divisa entre os Estados do Pará e do Maranhão. Fares e colaboradores comentam ainda sobre uma pesquisa realizada em 1999 por Barros, em que o autor registrou as constelações criadas por essa comunidade, que podem ser uma importante fonte de conhecimento acerca da organização socioeconômica e da cultura dos indígenas.

\section{A astronomia indígena na formação de professores: uma possibilidade a partir da abordagem CTS}

Diante do exposto, alguns autores como Afonso (2009) ressaltam o valor pedagógico do ensino da astronomia indígena para os alunos do ensino fundamental de todo o Brasil.

Para o autor, a validade desse conhecimento pauta-se no fato de se tratar de uma astronomia baseada em elementos sensoriais, e não em elementos geométricos e abstratos, e também por fazer alusão a elementos da nossa natureza (sobretudo fauna e flora) e história, promovendo autoestima e valorização dos saberes antigos, salientando que as diferentes interpretações da mesma região do céu, feitas por diversas culturas, auxiliam na compreensão das diversidades culturais, dentre elas a Ciência de modo mais amplo.

Nesse sentido, podemos mencionar o trabalho de Araújo (2014) que elaborou sequências didáticas para o Ensino Médio usando a Astronomia indígena como principal conhecimento a ser trabalhado. Com sua 
proposta, o autor pretendia estudar, por meio da pedagogia dialógica de Paulo Freire, o céu com o olhar da cultura indígena e não somente aquele conteúdo ligado à tradição greco-romana.

Por fim, Araújo (2014) reconhece a importância do ensino de uma cultura diferente (Astronomia indígena e não somente a grecoromana) para a promoção da autonomia e criticidade dos estudantes frente ao conhecimento.

Em conformidade com esses autores, a perspectiva CTS considera possível perceber o universo numa dimensão relativa, ou seja, que respeita a pluralidade cultural e que envolve a construção social da realidade e a consequente necessidade de respeitar as diferenças que daí emergem, observando a perspectiva humana e não somente técnica da astronomia.

Correa e Bazzo (2013) se apoiam em Maia e Monteiro ao afirmarem que aquilo que o enfoque CTS defende, "é o tratamento de questões científicas de forma que o estudante valorize este conhecimento por relacioná-lo a seus saberes cotidianos. Esta atitude possibilita a construção de uma abordagem crítica sobre a ciência, necessária para a construção da identidade cidadã" (MAIA; MONTEIRO, 2008, p. 3).

Dessa forma, concordamos com Fares et al. (2004, p. 78) quando eles defendem que as constelações, por exemplo, demonstram o quanto a subjetividade do olhar influenciado pelo contexto cultural é preponderante para a formação das estruturas sociais responsáveis pela elaboração e sistematização das diversas formas de conhecimentos que irão nortear a vida dos sujeitos sociais de uma dada sociedade. E ainda que, quando as pessoas olham para o céu e criam símbolos para resolver seus problemas cotidianos, ocorre aí a exteriorização de todo um universo cultural e imaginário, tendo em vista que as constelações, para quem as criou e para os povos que delas fazem uso, podem ser entendidas não só como um agrupamento de estrelas, mas como a representação simbólica de um conjunto de valores, crenças e costumes próprios de cada sociedade.

Por isso, é necessário pensar o mundo numa perspectiva relativa ou plural, de forma a propiciar o respeito às diferenças. Ou seja, o que Fares et al (2004) defendem é que a abordagem da astronomia indígena em sala de aula permitirá a construção de uma visão crítica sobre a cultura científica e a conscientização dos objetivos variados do conhecimento científico, de suas limitações e das bases sobre as quais se assentam suas asserções. Além disso, atestam que documentar e disseminar o conhecimento astronômico dos índios e de outros povos que correm o risco de desaparecer é importante para a preservação de um patrimônio de valor inestimável e a valorização da memória brasileira.

Corroborando Fares et al. (2004), Araújo (2014) defende que o ensino da Astronomia indígena favorece o desenvolvimento de um estudante crítico, pois ao levar para sala de aula uma visão distinta do que normalmente se usa para falar do assunto, os estudantes desenvolvem sua criticidade para compreender que a Ciência é uma construção humana e, como tal, tem suas limitações, seus períodos de crise, de afirmação e de controvérsias. Nesse sentido, a discussão sobre a Astronomia indígena favorece esse tipo de discussão em sala de aula, pois traz para os estudantes uma cultura rica e cheia de saberes.

Vemos então que o uso de elementos da Astronomia indígena, além do respeito à diversidade, pode colaborar no desenvolvimento da criticidade dos estudantes (FARES et al., 2004; AFONSO, 2009; ARAÚJO, 2014). Mas para isso temos que primeiramente inserir efetivamente esses conteúdos nos cursos de formação de professores, tanto do Ensino Fundamental, quanto do Médio (LANGHI, 2009).

A partir deste ponto, a valorização da cultura indígena pode então ser uma importante ferramenta na relação didática em sala de aula. Acreditamos que uma concepção filosófica de caráter interdisciplinar, como o enfoque CTS, pode auxiliar no rompimento dos moldes atuais, contribuindo para uma formação mais globalizadora e duradoura do conhecimento (BAZZO et al., 2008).

\section{Conclusões}

O presente artigo buscou discutir alguns aspectos referentes à valorização da cultura científica dos povos indígenas, bem como a importância dela para a promoção do pensamento crítico por parte dos estudantes e professores em formação. 
Entre as várias constatações oriundas do estudo em questão e apontadas no decorrer deste artigo, algumas merecem atenção especial.

A primeira é a que se refere à importância da etnociência e, especialmente, da astronomia indígena, para a formação cultural e o desenvolvimento das civilizações. A segunda e mais relevante é a necessidade de incluir ou reforçar a etnociência, a partir de uma abordagem CTS, nos cursos de formação de professores visando ao fortalecimento da sua criticidade e da sua reflexão.

Não queremos dizer com isso que o modelo vigente acontece de forma acrítica, imediatista ou descontextualizada. Apenas queremos ressaltar que o aluno pode transformar-se num agente de transformação social, capaz de refletir sobre assuntos aparentemente tão distantes, mas essencialmente tão próximos de sua realidade.

Com a ampliação de sua gama de conhecimentos e pontos de vista, os estudantes e, sobretudo, os professores em formação poderão compreender a legitimidade de diferentes modos de abordar os fenômenos naturais, permitindo-se realizar escolhas em relação àquilo que consideram importante e aplicar os conhecimentos que têm ao seu dispor (científicos e/ou tradicionais) nos contextos em que julgarem necessários e/ou apropriados (BAPTISTA, 2010).

Ao criar condições para o desenvolvimento da capacidade de conhecer, criticar, gerenciar, julgar e agir, mediante o exame e a discussão de problemas da vida cotidiana e da história das civilizações, a abordagem CTS pode contribuir para a formação de professores capazes de aplicar, de fato, os conhecimentos e atitudes adquiridos em sua prática pedagógica em questões de sua própria vida diária e, consequentemente, na construção de um mundo mais ético e mais humano.

\section{Referências}

AFONSO, G. B. Astronomia indígena. Anais da 61ํㅡㄹ Reunião Anual da SBPC - Manaus, 2009.

Disponível As constelações indígenas brasileiras.

$<$ http://www.telescopiosnaescola.pro.br/indigena s.pdf >. Acesso em: 05 fev. 2015.
ARAÚJO, D. C. C. de. Uma proposta para a inserção de tópicos de astronomia indígena brasileira no ensino médio: desafios e possibilidades. 2014. 185 fl. Dissertação (Mestrado profissional em Ensino de Ciências) UNB. Brasília, 2014.

BAPTISTA, G. C. S. Importância da demarcação de saberes no ensino de Ciências para sociedades tradicionais. Ciênc. educ. (Bauru), Bauru, v. 16, n. 3, 2010.2 Disponível em: $<$ http://www.scielo.br/scielo.php?script=sci_artte xt\&pid=S1516-

73132010000300012\&lng=en\&nrm=iso>. Acesso em: 9 fev. 2015.

BAZZO, W.A. et al. Educação tecnológica: enfoques para o ensino de engenharia. Florianópolis: Ed. da UFSC, 2008.

CHASSOT, A. I. Alfabetização Científica: questões e desafios para a educação. Ijuí: Unijuí, p. 191-230, 2001.

CHASSOT, A. I. Haciendo educación em ciências em los studios de Pedagogia com la inclusion de saberes populares em el curriculum. Memórias do Ciaem XII. [2007]. Disponível em: < http://ciaemiacme.org/memorias/xii_ciaem/123_estudios_ped agogia.pdf $>$. Acesso em: 05 de fev. 2015.

COBERN, W. W.; LOVING, C. C. Defining "Science" in a multicultural world: Implications for science education. Science Education, v. 85, p. 50-67, 2001.

CORREA, L.F.; BAZZO, W.A. A interdisciplinaridade efetiva requer 0 rompimento das fronteiras na Educação em Engenharia. Anais: XLI Congresso Brasileiro de Educação em Engenharia. Gramado-RS, 2013.

FARES, E. et al. O Universo das sociedades numa perspectiva relativa: exercícios da Etnoastronomia. Revista Latino-Americana de Educação em Astronomia - RELEA. n. 1, p. 7785, 2004.

FONSECA, O.; PINTO, S. P.; JURBERG, C. Mitos e constelações indígenas, confeccionando um planetário de mão. In X Reunón de la Red de Popularización de la Ciencia y la Tecnologia en 
América Latina y el Caribe y IV Taller "Ciencia, Comunicación y Sociedad". San José-Costa Rica, 2007.

FRANCISCO, Z. L. A educação informal e a educação formal: interfaces e significados de saberes no ensino de Química em Moçambique. Olhar de professor, v.8, n. 1, p. 141-150, 2005.

GEORGE, J. Science Teachers as Innovators using Indigenous Resources. International Journal of Science Education, v. 14, n. 1, p. 95 109, 1992.

HOFSTEIN, A., AIKENHEAD, G., RIQUARTS, K. Discussions over STS at the fourth IOSTE symposium. International Journal of Science Education, v. 10, n. 4, p.357-366, 1988.

LANGHI, R. Astronomia nos anos iniciais do ensino fundamental: repensando a formação de professores. 2009. 372 fl. Tese (Doutorado em Educação para a Ciência) - UNESP. Bauru, 2009.

LIMA, F. P.; MOREIRA, I. de C. Tradições astronômicas tupinambás na visão de Claude D'Abbeville. REVISTA DA SBHC. v. 3, n. 1, p. 4-19, 2005.

LIMA, A.C.S. Um olhar sobre a presença das populações nativas na invenção do Brasil. In: Silva, A. L. e Grupioni, L. D. B. (Org.). A Temática Indígena na Escola - Novos desafios para professores de $1^{\mathrm{o}}$ e $2^{\underline{o}}$ graus. 3 ed., São Paulo: Global, Brasília: MEC: MARI: UNESCO, 2000.

MAIA, D. P.; MONTEIRO, I. B. CTS Como Instrumento Para Formação Docente. In: $1^{0}$ Seminário Nacional de Educação Profissional e Tecnológica, 2008, Belo Horizonte - MG. Anais do primeiro seminário de educação profissional e tecnológica, 2008. Disponível em: $<$ http://www.senept.cefetmg.br/galerias/Arquivo s_senept/anais/terca_tema3/TerxaTema3Poster3. pdf $>$. Acesso em: 12 out. 2015.

PINHEIRO, P. C.; GIORDAN, M. O preparo do sabão de cinzas em Minas Gerais, Brasil: do status de etnociência à sua mediação para a sala de aula utilizando um sistema hipermídia etnográfico. Investigações em Ensino de Ciências, v. 15, n. 2, p. 355-383, 2010.
SANTOS, W. L. P.; MORTIMER, E. F. Uma análise de pressupostos teóricos da abordagem C-T-S (Ciência-Tecnologia-Sociedade) no contexto da educação brasileira. Ensaio Pesquisa em Educação em Ciências, v.2, n.2, 2002.

SILVA, S. F. da. Sistema de numeração dos Guarani: caminhos para a prática pedagógica. 2011. Dissertação (Mestrado em Educação Científica e Tecnológica) - Universidade Federal de Santa Catarina. Florianópolis, 2011.

TRÉZ, T. de A. e. Feyerabend, interculturalismo e etnobiologia: algumas possíveis articulações no ensino de Biologia. Biotemas, v. 24, n. 3, p. 129140, 2011. 